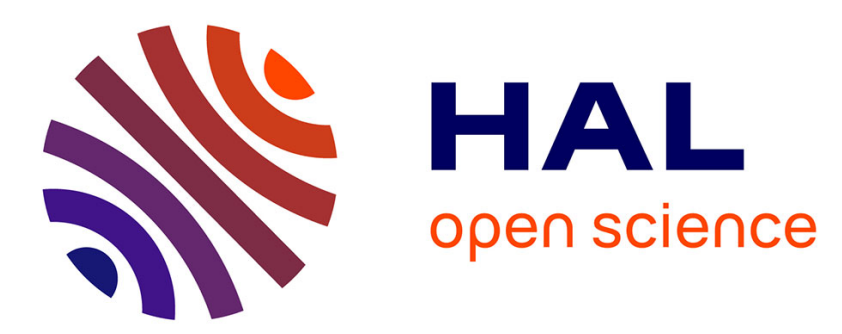

\title{
Euclidean Distance Decomposition to Generate New 16-QAM and 64-QAM Space-Time Trellis Codes
}

Pierre Viland, Gheorghe I. Zaharia, Jean-François Hélard

\section{To cite this version:}

Pierre Viland, Gheorghe I. Zaharia, Jean-François Hélard. Euclidean Distance Decomposition to Generate New 16-QAM and 64-QAM Space-Time Trellis Codes. PIMRC, Sep 2010, Istambul, Turkey. pp.1-5. hal-00542003

\section{HAL Id: hal-00542003 https://hal.science/hal-00542003}

Submitted on 3 Dec 2010

HAL is a multi-disciplinary open access archive for the deposit and dissemination of scientific research documents, whether they are published or not. The documents may come from teaching and research institutions in France or abroad, or from public or private research centers.
L'archive ouverte pluridisciplinaire HAL, est destinée au dépôt et à la diffusion de documents scientifiques de niveau recherche, publiés ou non, émanant des établissements d'enseignement et de recherche français ou étrangers, des laboratoires publics ou privés. 


\title{
Euclidean Distance Decomposition to Generate New 16-QAM and 64-QAM Space-Time Trellis Codes
}

\author{
Pierre Viland, Gheorghe Zaharia and Jean-François Hélard \\ Institute for Electronics and Telecommunications of Rennes - UMR CNRS 6164 \\ INSA - 20 avenue des Buttes de Coesmes- CS 70839 \\ 35043 Rennes Cedex 7- France \\ Email: pierre.viland@insa-rennes.fr
}

\begin{abstract}
In this paper, the generation of 16-QAM and 64-QAM space-time trellis codes (STTCs) for several transmit antennas is considered. The main problem with an exhaustive search is the important time to find the best $2^{2 n}$-QAM STTCs, especially for great values of $n$ and for great numbers of transmit antennas. In order to reduce this search time, an efficient method must be used to generate optimal $2^{2 n}$-QAM STTCs. Thus, a new method based on the decomposition of the Euclidean distance between 2 codewords is proposed to design the optimal STTCs. Thanks to this new method, the first 16-QAM STTCs with more than 2 transmit antennas and the first 64-QAM STTCs with 2 and 3 transmit antennas are proposed. The performance of these new codes is evaluated by simulation.
\end{abstract}

\section{INTRODUCTION}

Introduced by Tarokh et al. in [1], space-time trellis codes (STTCs) reduce the detrimental effects of fading in order to improve the error performance. STTCs use trellis-coded modulations (TCMs) over multiple input multiple output (MIMO) channels via several transmit and receive antennas. Thus, STTCs combine diversity gain and coding gain leading to a reduction of the error probability.

In the case of slow fading Rayleigh channels, the rank and determinant criteria are proposed in [1] to reduce the pairwise error probability (PEP) of STTCs. In [2], the Euclidean distance (ED) between two codewords is revealed to strengthen the design of STTCs. This ED criterion is used to construct 4-PSK and 8-PSK STTCs when the product $n_{T} n_{R}$ is large [3], [4] where $n_{T}$ and $n_{R}$ are the number of transmit and receive antennas respectively. In [5], Liao et al. show that the code performance is also governed by the union bound which is a function of PEP and the distance spectrum [6]. The distance spectrum shows the weight repartition of either the determinants for a small product $n_{T} n_{R}$ or the EDs between two codewords for a great product $n_{T} n_{R}$.

In [7], Lui et al. propose the $\Sigma_{0}$ criterion, which is a specific rank criterion to design $2^{2 n}$-QAM STTCs with $n \in \mathbb{N}^{*}$. New 16-QAM STTCs with improved performance are presented in [8], governed by both $\Sigma_{0}$ criterion and trace criterion. To design the best STTCs, Hong et al. [9] consider the union bound with the distance spectrum computed for the determinants. Regrettably, these new 16-QAM STTCs are given with only 2 transmit antennas because the exhaustive search for 16-QAM STTCs with more than 2 transmit antennas requires a long search time.
The focus of this paper is to propose a method to generate $2^{2 n}$-QAM STTCs such as 16/64-QAM STTCs which reduces the search time compared to the exhaustive search. This method is based on the decomposition of the ED between 2 codewords and the partition of the generator matrix of the code into blocks. Each term of the ED is computed via specific blocks of the generator matrix. The search method consists in selecting blocks which increase the terms of ED between 2 codewords.

The rest of the paper is organized as follows. The system model is given in section II. The design criteria of $2^{2 n}$-QAM STTCs are reviewed in section III. Section IV describes the method used to create new 16/64-QAM STTCs which are presented in section V. The performance of these new STTCs is given in section VI.

\section{SYSTEM MODEL}

We consider a $2^{2 n}$-QAM $4^{n \nu}$ states space-time trellis encoder with $n_{T}$ transmit antennas, as proposed in [7]-[9]. For $n=2$, i.e. for a 16-QAM, this encoder is shown in Fig. 1.

For a $2^{2 n}$-QAM STTC, at each time $t \in \mathbb{Z}$, the input mapper converts the $2 n$ input bits $b_{1}^{t} \cdots b_{n}^{t}$ and $b_{n+1}^{t} \cdots b_{2 n}^{t}$ into 2 input symbols $x_{1}^{t} \in \mathbb{Z}_{2^{n}}$ and $x_{2}^{t} \in \mathbb{Z}_{2^{n}}$ via the natural mapping, where $b_{n}^{t}$ and $b_{2 n}^{t}$ are the least significant bits (LSB). The input block containing these two $\mathbb{Z}_{2^{n}}$ input symbols is followed by $\nu$ memory blocks of the same length. Each memory block contains two memory-cells for two $\mathbb{Z}_{2^{n}}$ symbols. A state at time $t$ is defined by the values of the $2 \nu$ symbols of the memory blocks. At each time $t$, the two $\mathbb{Z}_{2^{n}}$ symbols of each block are replaced by the two $\mathbb{Z}_{2^{n}}$ symbols of the previous block. For each block $i, 1 \leq i \leq \nu+1$, the $l^{\text {th }}$ symbol with $l \in\{1,2\}$ is associated to $n_{T}$ complex coefficients $g_{l, i}^{k}=g_{l, i}^{k, I}+j g_{l, i}^{k, Q} \in \mathbb{Z}_{2^{n}}(j)$ where $\mathbb{Z}_{2^{n}}(j)$ is the complex ring of the integers modulo $2^{n}$ and $1 \leq k \leq n_{T}$. With these $n_{T} \times 2(\nu+1)$ complex coefficients, the generator matrix $G$ is obtained

$$
\begin{aligned}
\boldsymbol{G}= & \boldsymbol{G}_{I}+j \boldsymbol{G}_{Q} \\
= & {\left[G_{1}^{1, I} G_{2}^{1, I}|\cdots| G_{1}^{\nu+1, I} G_{2}^{\nu+1, I}\right] } \\
& +j\left[G_{1}^{1, Q} G_{2}^{1, Q}|\cdots| G_{1}^{\nu+1, Q} G_{2}^{\nu+1, Q}\right],
\end{aligned}
$$

with $G_{l}^{i, I}=\left[g_{l, i}^{1, I} \cdots g_{l, i}^{n_{T}, I}\right]^{\mathrm{T}} \in \mathbb{Z}_{2^{n}}^{n_{T}}$ and $G_{l}^{i, Q}=$ 


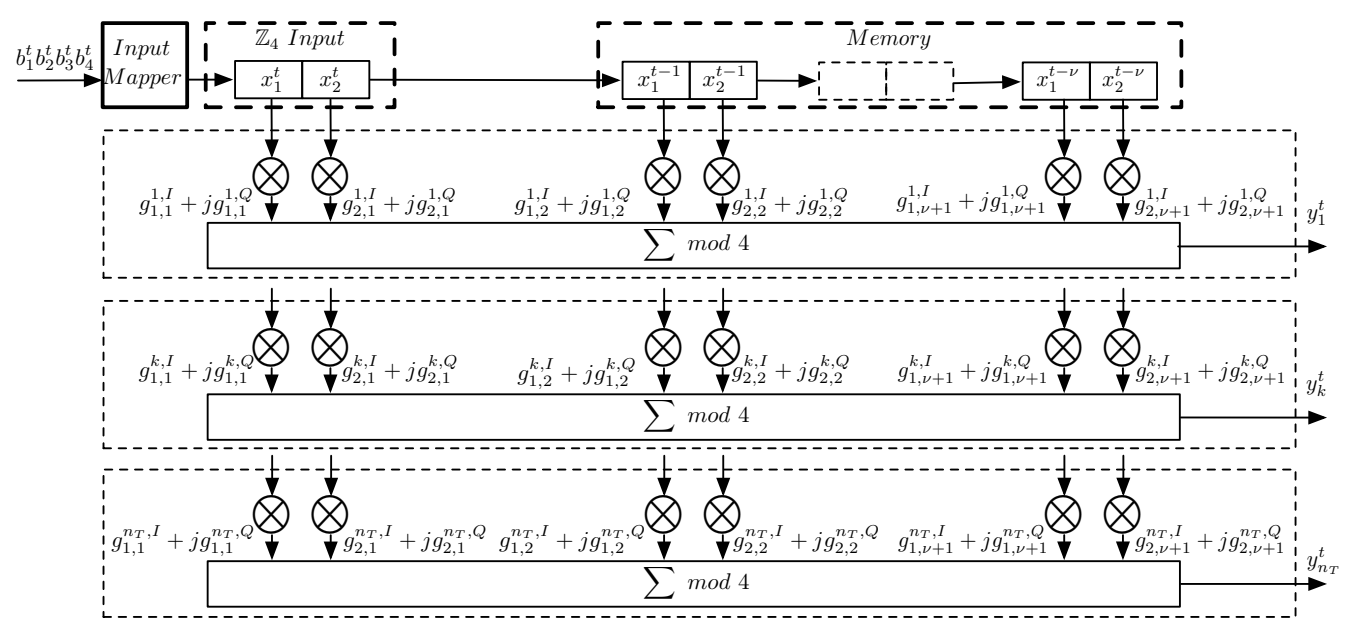

Fig. 1. 16-QAM $4^{2 \nu}$ states space-time trellis encoder with $n_{T}$ transmit antennas.

$\left[g_{l, i}^{1, Q} \cdots g_{l, i}^{n_{T}, Q}\right]^{\mathrm{T}} \in \mathbb{Z}_{2^{n}}^{n_{T}}$. In this paper, the matrix $V^{\mathrm{T}}$ represents the transpose of $V$.

At each time $t$, the encoder output $Y_{X^{t}}=\left[y_{1}^{t} y_{2}^{t} \cdots y_{n_{T}}^{t}\right]^{\mathrm{T}} \in$ $\mathbb{Z}_{2^{n}}^{n_{T}}(j)$ is given by

$$
\begin{aligned}
Y_{X^{t}} & =\boldsymbol{G} X^{t} \bmod 2^{n} \\
& =\boldsymbol{G}_{I} X^{t}+j \boldsymbol{G}_{Q} X^{t} \bmod 2^{n},
\end{aligned}
$$

where $X^{t}=\left[x_{1}^{t} x_{2}^{t}|\cdots| x_{1}^{t-\nu} x_{2}^{t-\nu}\right]^{\mathrm{T}} \in \mathbb{Z}_{2^{n}}^{2(\nu+1)}$ is the extended-state at time $t$ defined by the two $\mathbb{Z}_{2^{n}}$ symbols of the input block and the $\nu$ memory blocks.

At time $t$, the $2^{2 n}$-QAM signal $s_{k}^{t}$ of the $k^{t h}$ transmit antenna is given by the mapping function $s_{k}^{t}=\Phi\left(y_{k}^{t}\right)$ defined by

$$
\begin{aligned}
\Phi: & \mathbb{Z}_{2^{n}}(j) \rightarrow \mathbb{C} \\
& s_{k}^{t}=y_{k}^{t}-\frac{2^{n}-1}{2}(1+j) .
\end{aligned}
$$

Finally, each output signal $s_{k}^{t}$ is send by the $k^{t h}$ transmit antenna. The MIMO signals transmitted simultaneously over the fading MIMO channel are given by $S^{t}=\left[s_{1}^{t} \cdots s_{n_{T}}^{t}\right]^{\mathrm{T}}$ and the column matrix $R^{t}=\left[r_{1}^{t} \cdots r_{n_{R}}^{t}\right]^{\mathrm{T}}$ of the receive MIMO signals with $n_{R}$ the number receive antennas can be written as

$$
R^{t}=\boldsymbol{H}^{t} S^{t}+N^{t},
$$

where $N^{t}=\left[n_{1}^{t} \cdots n_{n_{R}}^{t}\right]^{\mathrm{T}}$ is a column matrix of complex additive white gaussian noises (AWGN) at time $t$ and $\boldsymbol{H}^{t}$ is a $n_{R} \times n_{T}$ matrix representing the complex path gains of the MIMO channel between the transmit and receive antennas.

\section{DESIGN CRITERIA}

The main design criteria have been established in [1]-[3] in order to decrease the bit and frame error rates. In this paper, only the case of slow fading channels is considered, i.e. the fading coefficients within each frame are constant. Besides, we assume that the decoder uses a maximum likelihood algorithm to estimate the transmitted signals.
The main goal of this design is to reduce the PEP which is the probability that the decoder selects an erroneous frame. It is possible to represent a codeword of $L$ MIMO signals starting at $t=0$ by a $n_{T} \times L$ matrix $S=\left[S^{1} S^{2} . . S^{L}\right]$ where $S^{t}$ is the $t^{t h}$ MIMO signal. An error occurs if the decoder decides that another codeword $\boldsymbol{E}=\left[E^{1} E^{2} \ldots E^{L}\right]$ is transmitted.

Let define the $n_{T} \times L$ difference matrix $\boldsymbol{B}=\boldsymbol{E}-\boldsymbol{S}$ :

$$
\boldsymbol{B}=\left[\begin{array}{ccc}
e_{1}^{1}-s_{1}^{1} & \ldots & e_{1}^{L}-s_{1}^{L} \\
\vdots & \ddots & \vdots \\
e_{n_{T}}^{1}-s_{n_{T}}^{1} & \ldots & e_{n_{T}}^{L}-s_{n_{T}}^{L}
\end{array}\right]
$$

The $n_{T} \times n_{T}$ product matrix $\boldsymbol{A}=\boldsymbol{B} \boldsymbol{B}^{*}$ is introduced, where $\boldsymbol{B}^{*}$ denotes the hermitian of $\boldsymbol{B}$. We define $r=$ $\min (\operatorname{rank}(\boldsymbol{B}))$, where $\boldsymbol{A}$ is computed for all pairs of coded frames $(\boldsymbol{E}, \boldsymbol{S})$. The design criteria depend on the value of the product $r n_{R}$.

$r n_{R} \leq 3$ : In this case, for a slow Rayleigh fading channel, two criteria have been proposed [1], [5] to reduce the PEP:

- $\boldsymbol{A}$ has to be a full rank matrix for any pair $(\boldsymbol{E}, \boldsymbol{S})$.

- The coding gain is given by $\eta=\sum_{d} N(d) d^{-n_{R}}$, where $N(d)$ is defined as the average number of error events with determinant $d=\operatorname{det}(\boldsymbol{A})$. The best codes must have the minimum value of $\eta$.

$r n_{R} \geq 4$ : In [3], based on [2], it is shown that for a large value of $r n_{R}$ which corresponds to a large number of independent SISO channels, the PEP is minimized if the sum of all the eigenvalues of the matrix $A$ is maximized. Since $A$ is a square matrix, the sum of all the eigenvalues is equal to the trace of the matrix $A$ given by

$$
\operatorname{tr}(\boldsymbol{A})=\sum_{t=1}^{L} d_{E}^{2}\left(E^{t}, S^{t}\right)
$$

where $d_{E}^{2}\left(E^{t}, S^{t}\right)=\sum_{k=1}^{n_{T}}\left|e_{k}^{t}-s_{k}^{t}\right|^{2}$ is the ED between the MIMO signals $E^{t}$ and $\bar{S}^{t}$ at time $t$.

For each pair of codewords, $\operatorname{tr}(\boldsymbol{A})$ is computed. The minimum trace is the minimum of all these values $\operatorname{tr}(\boldsymbol{A})$. The 
minimization of the PEP amounts to using a code which has the maximum value of the minimum trace. In other words, the EDs between two paths of the trellis must be maximized. In [5], it is also stated that to minimize the frame error rate (FER), the number of error events with minimum EDs (or trace) has to be minimized.

In this paper, we consider the case $r n_{R} \geq 4$ which is obtained when the rank of STTCs is greater than 1 and there are at least 2 receive antennas.

\section{Codes Design VIA THE EDS DECOMPosition}

Since the number of $2^{2 n}$-QAM STTCs is large, an efficient method to design the best codes must allow to decrease the search time compared to the exhaustive search. The proposed method is based on the decomposition of EDs between two different codewords.

In the next sections, the ED between 2 codewords is notified by 'Cumulated ED' (CED), in opposition with the ED between two MIMO signals.

\section{A. Preliminary}

This section gives tools and definitions to design STTCs via the EDs decomposition. The ED criterion is based on the maximization of the minimum CED. At each time $t$, the CED is the sum of the Euclidian distances between the MIMO signals of two different codewords.

To compute the CEDs, two conditions must be reminded:

- Initial condition: the initial state of the encoder must be equal to $W^{0}=[00|\cdots| 00]$.

- Final condition: the final state of the encoder must be equal to $W^{L+1}=[00|\cdots| 00]$.

For a $2^{2 n}$-QAM $2^{2 n \nu}$ states STTC with $n_{T}$ transmit antennas, two different input sequences of $2(L-\nu) \mathbb{Z}_{2^{n}}$ symbols given by the input mapper, as shown in Fig. 1, are considered:

- $X_{e}=\left[x_{e, 1}^{1} x_{e, 2}^{1}\left|x_{e, 1}^{2} x_{e, 2}^{2}\right| \cdots \mid x_{e, 1}^{L-\nu} x_{e, 2}^{L-\nu}\right]$

- $X_{s}=\left[x_{s, 1}^{1} x_{s, 2}^{1}\left|x_{s, 1}^{2} x_{s, 2}^{2}\right| \cdots \mid x_{s, 1}^{L-\nu} x_{s, 2}^{L-\nu}\right]$

These two sequences generate two codewords $\boldsymbol{E}$ and $\boldsymbol{S}$ of length $L$. These sequences correspond to two different paths in the trellis.

At each time $t \leq L$, the two symbols $x_{e, 1}^{t} x_{e, 2}^{t}$ and $x_{s, 1}^{t} x_{s, 2}^{t}$ of the sequences $X_{e}$ and $X_{s}$ feeded into the encoder supply the two MIMO signals $S^{t}=\left[s_{1}^{t} \cdots s_{n_{T}}^{t}\right]$ and $E^{t}=\left[e_{1}^{t} \cdots e_{n_{T}}^{t}\right]$ given by

$$
\begin{aligned}
E^{t} & =\Phi\left(\boldsymbol{G} X_{e}^{t} \bmod \left(2^{n}\right)\right) \\
S^{t} & =\Phi\left(\boldsymbol{G} X_{s}^{t} \bmod \left(2^{n}\right)\right),
\end{aligned}
$$

where $X_{e}^{t}=\left[x_{e, 1}^{t} x_{e, 2}^{t}|\ldots| x_{e, 1}^{t-\nu} x_{e, 2}^{t-\nu}\right]$ and $X_{s}^{t}=$ $\left[x_{s, 1}^{t} x_{s, 2}^{t}|\ldots| x_{s, 1}^{t-\nu} x_{s, 2}^{t-\nu}\right]$ are the extended-states at time $t$ corresponding to the two sequences $X_{e}$ and $X_{s}$ respectively.

The ED between two corresponding MIMO signals at time $t$ is given by $d_{E}^{2}\left(E^{t}, S^{t}\right)=\sum_{k=1}^{n_{T}}\left|e_{k}^{t}-s_{k}^{t}\right|^{2}$.
It is possible to compute this ED thanks to the two corresponding extended-states $X_{e}^{t}$ and $X_{s}^{t}$ and the generator matrix $G$ via the function $D_{E}$ defined as

$$
\begin{aligned}
& D_{E}: \mathbb{Z}_{2^{n}}^{2(\nu+1)} \times \mathbb{Z}_{2^{n}}^{2(\nu+1)} \rightarrow \mathbb{R}^{+} \\
& D_{E}\left(X_{e}^{t}, X_{s}^{t}\right)=d_{E}^{2}\left(\Phi\left(\boldsymbol{G} X_{e}^{t}\right), \Phi\left(\boldsymbol{G} X_{s}^{t}\right)\right) .
\end{aligned}
$$

Thus, each CED is given by

$$
C E D\left(X_{e}, X_{s}\right)=\sum_{t=1}^{L} D_{E}\left(X_{e}^{t}, X_{s}^{t}\right) \text {. }
$$

The first term of each CED is $D_{E}\left(X_{e}^{1}, X_{s}^{1}\right)$ with

$$
\begin{aligned}
X_{e}^{1} & =\left[x_{e, 1}^{1} x_{e, 2}^{1}|00| \ldots \mid 00\right] \\
X_{s}^{1} & =\left[x_{s, 1}^{1} x_{s, 2}^{1}|00| \ldots \mid 00\right]
\end{aligned}
$$

and the last term is $D_{E}\left(X_{e}^{L}, X_{s}^{L}\right)$ with

$$
\begin{aligned}
X_{e}^{L} & =\left[00|\ldots| 00 \mid x_{e, 1}^{L-\nu} x_{e, 2}^{L-\nu}\right] \\
X_{s}^{L} & =\left[00|\ldots| 00 \mid x_{s, 1}^{L-\nu} x_{s, 2}^{L-\nu}\right]
\end{aligned}
$$

Besides, it is easy to show that the $m^{\text {th }}$ first term and the $(L-m+1)^{t h}$ last term of CEDs depend of the $m$ first blocks and the $m$ last blocks of $G$ respectively for $m \leq \nu+1$.

Let us consider the case of $2^{n(\nu+1)}$ states $2^{2 n}$-QAM STTCs. if

- $\nu$ is odd, we define $\alpha_{F}=\frac{\nu+1}{2}$ and $\alpha_{L}=\alpha_{F}-1$.

- $\nu$ is even, we define $\alpha_{F}=\frac{\nu}{2}$ and $\alpha_{L}=\alpha_{F}$.

To ensure that the CEDs of STTCs are maximized, the sum of $\alpha_{F}$ first terms

$$
\sum_{t=1}^{\alpha_{F}} D_{E}\left(X_{e}^{t}, X_{s}^{t}\right)
$$

must be maximized for all pairs $\left(X_{e}^{t}, X_{s}^{t}\right)$ via the selection of the $\alpha_{F}$ first blocks.

In the same way and independently of the sum of the $\alpha_{F}$ first terms, the sum of the $\alpha_{L}$ last terms

$$
\sum_{t=L-\alpha_{L}+1}^{L} D_{E}\left(X_{e}^{t}, X_{s}^{t}\right)
$$

must be maximized for all pairs $\left(X_{e}^{t}, X_{s}^{t}\right)$ via the selection of the $\alpha_{L}$ last blocks. The $\left(\alpha_{F}+1\right)^{t h}$ term must be selected to maximized the CED and create a generator matrix with a rank greater than 1 in function of the previous selections.

In these two case ( $\nu$ is odd or even), the set of the $\alpha_{F}$ first blocks is called $\mathcal{B}_{F}$ and the set of the $\alpha_{L}$ blocs is called $\mathcal{B}_{M}$. No block of $G$ belongs to both $\mathcal{B}_{F}=\left\{\left[G_{1}^{1} \cdots G_{n}^{1}\right], \cdots,\left[G_{1}^{\alpha_{F}} \cdots G_{n}^{\alpha_{F}}\right]\right\}$ and $\mathcal{B}_{L}=$ $\left\{\left[G_{1}^{\alpha_{L}+1} \cdots G_{n}^{\alpha_{L}+1}\right], \cdots,\left[G_{1}^{\nu+1} \cdots G_{n}^{\nu+1}\right]\right\}$ i.e.

$$
\mathcal{B}_{F} \bigcap \mathcal{B}_{L}=\emptyset \text {. }
$$

Thus, the $\alpha_{F}$ first terms and the $\alpha_{L}$ last terms are totally independent. The $\left(\alpha_{F}+1\right)^{t h}$ block creates the dependance of these sets of terms in order to maximize the CED.

Remark : If $\nu=1, \alpha_{L}=0$. In this case, the $\left(\alpha_{F}+1\right)^{t h}$ block must be maximized the last term independently of the first block. 


\section{B. The EDs decomposition}

To assure that the CED between two $\mathbb{Z}_{2^{n}}$ sequences $X_{e}=$ $\left[x_{e, 1}^{1} x_{e, 2}^{1}|\ldots| x_{e, 1}^{L-1} x_{e, 2}^{L-1}\right]$ and $X_{s}=\left[x_{s, 1}^{1} x_{s, 2}^{1}|\ldots| x_{s, 1}^{L-1} x_{s, 2}^{L-1}\right]$ is maximized, we proceed as follows.

The minimum result of the sum of $\alpha_{F}$ first terms of the CED

$$
\sum_{t=1}^{\alpha_{F}} D_{E}\left(X_{e}^{t}, X_{s}^{t}\right)
$$

must be maximized for all pairs $\left(X_{e}^{t}, X_{s}^{t}\right)$. Therefore, The $\alpha_{F}$ first blocks must be selected as followed:

- The first block used to compute the first term $D_{E}\left(X_{e}^{1}, X_{s}^{1}\right)$ must be selected to generate a subset of $\mathbb{Z}_{2^{n}}^{n_{T}}(j)$ containing the MIMO symbols separated by the largest EDs.

- if $i$ blocks have been already selected with $1 \leq i \leq$ $\alpha_{F}-1$, the $(i+1)^{t h}$ block must be selected to maximized $\sum_{t=1}^{i+1} D_{E}\left(X_{e}^{t}, X_{s}^{t}\right)$.

In the same way, The minimum result of the sum of the $\alpha_{L}$ last terms

$$
\sum_{t=L-\alpha_{L}+1}^{L} D_{E}\left(X_{e}^{t}, X_{s}^{t}\right)
$$

must be maximized for all pairs $\left(X_{e}^{t}, X_{s}^{t}\right)$. Therefore, the $\alpha_{L}$ last blocks must be selected as followed:

- The last block used to compute the last term $D_{E}\left(X_{e}^{L}, X_{s}^{L}\right)$ must be selected to generated a subset of $\mathbb{Z}_{2^{n}}^{n_{T}}(j)$ containing the MIMO symbols separated by the largest EDs.

- if $i$ blocks have been already selected with $1 \leq i \leq \alpha_{L}-$ 1 , the $(i+1)^{t h}$ last block must be selected to maximized $\sum_{t=i}^{L} D_{E}\left(X_{e}^{t}, X_{s}^{t}\right)$.

Further on, the last block must generate a subset and must maximized the CED.

Remark: If the EDs decomposition is used to design a $4^{n}$-QAM STTC, the MIMO symbols originating from or merging into the same state are separated by the largest EDs. This principe is one of the rules of the set partitioning proposed by Ungerboeck to design TCMs [10].

\section{Example of the EDs decomposition for $2^{2 n}-Q A M, 4^{n}$ states STTCs}

The case of $2^{2 n}$-QAM $4^{n}$ states $(\nu=1)$ STTCs with $n_{T}$ transmit antennas is presented. The generator matrix $G$ is constituted by 2 blocks of 2 columns. Each column $G_{l}^{i}$ belongs to $\mathbb{Z}_{2^{n}}^{n_{T}}(j)$ for $i \in\{1,2\}$ and $l \in\{1,2\}$.

To find the best code, the first set is the generation of optimal block. In fact, each block $i$ of the generator matrix generates a set of MIMO symbols (which is a subgroup of $\left.\mathbb{Z}_{2^{n}}^{n_{T}}(j)\right)$ given by

$$
\Lambda_{i}=\left\{\sum_{l=1}^{2} x_{l} G_{l}^{i} \bmod 2^{n} / x_{l} \in \mathbb{Z}_{2^{n}}\right\} .
$$

The blocks with the best distance spectrum are called 'optimal blocks'. The distance spectrum of the block $i$ is the repartition of EDs between the elements of $\Lambda_{i}$.

For example, the minimal EDs between the elements of 16QAM optimal blocks with $n_{T}=2$ is 4 , this minimum EDs with $n_{T}=3$ is 8 and the minimum ED with $n_{T}=4$ is 12 . For the 64-QAM optimal blocks, the minimal EDs between the elements with $n_{T}=2$ is 9 and the minimum ED with $n_{T}=3$ is 22 . These EDs are given without normalization of the power.

After this generation of optimal blocks, the codes are designed by the combination of two different optimal blocks. Among this set of codes, the best STTCs are those with the best CEDs.

\section{Example of the EDs decomposition for $2^{2 n}-Q A M 16^{n}$ states STTCS}

The case of $2^{2 n}$-QAM $16^{n}$ states STTCs with $n_{T}$ transmit antennas is considered $(\nu=2)$. The generator matrix $G$ is constituted by 3 blocks of 2 columns. Each column $G_{l}^{i}$ belongs to $\mathbb{Z}_{2^{n}}^{n_{T}}(j)$ for $i \in\{1,2,3\}$ and $l \in\{1,2\}$.

The CED is computed between two $\mathbb{Z}_{2^{n}}$ sequences $\quad X_{e}=\left[x_{e, 1}^{1} x_{e, 2}^{1}|\cdots| x_{e, 1}^{L-2} x_{e, 2}^{L-2}\right] \quad$ and $X_{s}=\left[x_{s, 1}^{1} x_{s, 2}^{1}|\cdots| x_{s, 1}^{L-2} x_{s, 2}^{L-2}\right]$. The number of states being $16^{n}$, then $\alpha_{F}=1$ and $\alpha_{L}=1$.

To design $\boldsymbol{G}$, there are two steps:

- The first step is to select the elements of $B_{1}$ and $B_{3}$ used to compute the first and the last terms. To maximize the first and the last term, the first and the last blocks must be selected among the optimal blocks. The generation of optimal first and last blocks is identical to the generation of the optimal blocks for the $4^{n}$ states STTCs.

- The second step is the selection of the block $B_{2}$. Its columns must be selected to increase the minimal CED of the generated code.

The columns of each block can be permuted to obtain the codes with the best CED between two codewords.

\section{E. Usefulness of the EDs decomposition}

The first method used to design the $2^{2 n}$-QAM STTCs is called $\Sigma_{0}$ criterion [7]. Liu et al. exploit the linearity of codes to decrease the complexity to compute the minimum rank because this computation does not require to check the rank for all pairs of possible input sequences.

In [8] the codes are designed according to 3 steps:

- The set of codes is reduced by exploiting the symmetry of codes.

- Among this set of codes, the codes which achieve full diversity are obtained via the $\Sigma_{0}$ criterion.

- The ED criterion is applied within this new set of codes.

In the case of 16-QAM 16 states STTCs, the number of codes is $4^{16} \approx 4.295 \times 10^{9}$. Wong et al. [8] reduce to $9,3 \%$ the number of codes which must be analyzed via the ED criterion.

In [9], Hong et al. use the $\Sigma_{0}$ criterion to find the codes which achieve full diversity. Among these codes, the optimal 
design based on the union bound [5] is used to optain the best codes. In their paper, the time to generate the STTCs is slightly reduced.

With the proposed method and without the properties of symmetries, in the case of 16-QAM 16 states STTCs with 2 transmit antennas, there are 6912 optimal blocks. With the EDs decomposition, the generator is composed by two optimal blocks. Thus, it is sufficient to analyze only $6912^{2}$ codes i.e. $1.1 \%$ of the totality of codes.

In the case of 16-QAM 16 states STTCs, the number of optimal blocks is 264961 . The total number of generator matrices is about $2.8147 \times 10^{14}$. The number of codes created by the EDs decomposition is about $7.02 \times 10^{10}$ which correspond to $0.02 \%$ of the totality of codes. For the $4^{n}$-QAM $4^{n}$ states STTCs, the percentage of codes generated by the EDs decomposition decrease when the number of transmit antennas or/and $n$ increase.

Th percentage of 16-QAM 256 states STTCs designed with the EDs decomposition is the same that the percentage of 16-QAM 16 states STTCs. In fact, for the 16-QAM 256 states STTCs designed with the EDs decomposition, the first and the last block is selected belong to optimal block. The 'middle' block is selected only to maximized the CED without conditions.

\section{NEW CODES}

This section presents new codes and the known corresponding codes. For each code, the minimum trace is given without normalization. In Table I, the previous published codes and new codes are shown. The WYCK's code has been presented in [8]. The Hong's code has been presented in [9].

TABLE I

16-QAM STTCS WITH 2 TRANSMIT ANTENNAS

\begin{tabular}{|c|c|c|c|c|c|c|c|c|c|c|}
\hline States & Code & \multicolumn{7}{|c|}{$G$} & & Trace \\
\hline 16 & WYCK [8] & & $\begin{array}{c}\boldsymbol{G}_{I} \\
\boldsymbol{G}_{Q}\end{array}$ & $\begin{array}{l}= \\
=\end{array}$ & $\begin{array}{l}3 \\
0 \\
0 \\
2\end{array}$ & \begin{tabular}{l|l}
1 & $\mid$ \\
2 & \\
1 & $\mid$
\end{tabular} & $\begin{array}{l}2 \\
1 \\
1 \\
1\end{array}$ & $\begin{array}{l}0 \\
2 \\
0 \\
1\end{array}$ & & 12 \\
\hline 16 & Hong [9] & & $\begin{array}{c}\boldsymbol{G}_{I} \\
\boldsymbol{G}_{Q}\end{array}$ & $\begin{array}{l}= \\
=\end{array}$ & $\begin{array}{l}0 \\
1 \\
0 \\
2\end{array}$ & \begin{tabular}{l|}
2 \\
0 \\
1 \\
2
\end{tabular} & $\begin{array}{l}3 \\
0 \\
1 \\
2\end{array}$ & $\begin{array}{l}2 \\
2 \\
2 \\
1\end{array}$ & & 8 \\
\hline 16 & New 1 & & $\begin{array}{c}\boldsymbol{G}_{I} \\
\boldsymbol{G}_{Q}\end{array}$ & $\begin{array}{l}= \\
=\end{array}$ & 0 & $\begin{array}{l}1 \\
2 \\
2 \\
3\end{array}$ & 3 & $\begin{array}{l}2 \\
2 \\
1 \\
3\end{array}$ & & 12 \\
\hline 256 & New 2 & $\begin{array}{c}\boldsymbol{G}_{I} \\
\boldsymbol{G}_{Q}\end{array}$ & $\begin{array}{l}= \\
=\end{array}$ & 0 & $\begin{array}{l}1 \\
2\end{array}$ & $\begin{array}{l}1 \\
1 \\
1 \\
2\end{array}$ & $\begin{array}{l}2 \\
3 \\
0\end{array}$ & $\begin{array}{l}2 \\
3 \\
1 \\
1\end{array}$ & $\begin{array}{l}3 \\
3 \\
0\end{array}$ & 18 \\
\hline
\end{tabular}

Due to the complexity of the exhaustive search, no 16-QAM STTC has been proposed with more than 2 transmit antennas. Tables II and III show new proposed 16-QAM codes with 3 and 4 transmit antennas respectively. In Table IV, new 64QAM STTCs with 2 and 3 transmit antennas are given.

Despite the reduction of the number of analyzed codes, the number of remaining codes is important.
TABLE II

16-QAM STTCS WITH 3 TRANSMIT ANTENNAS

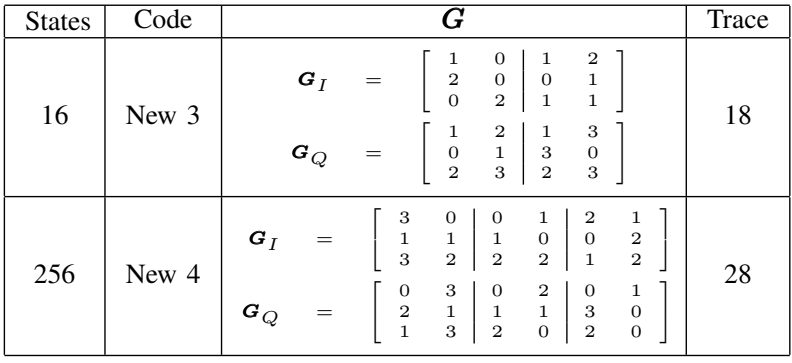

TABLE III

16-QAM 16 STATES STTCS WITH 4 TRANSMIT ANTENNAS

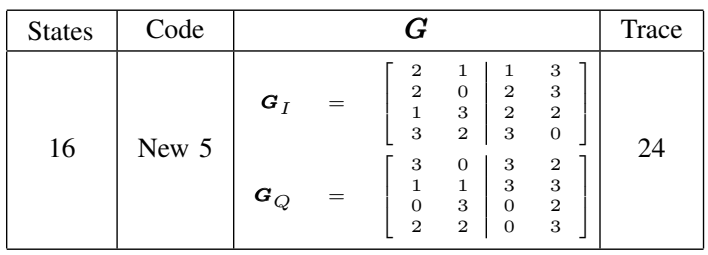

TABLE IV

64-QAM 64 STATES STTCS WITH 2 AND 3 TRANSMIT ANTENNAS

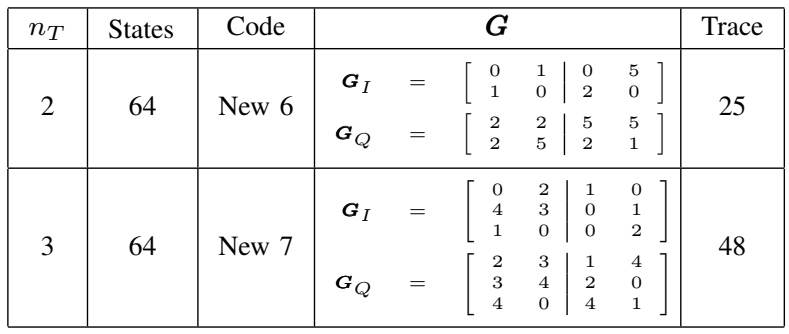

\section{Simulation RESUlts}

The performance of each code is evaluated by simulation in a slow Rayleigh fading channel. The channel fading coefficients are independent samples of a complex Gaussian process with zero mean and variance 0.5 per dimension. These channel coefficients are assumed to be known by the decoder. Each frame consists of 66 16-QAM or 64-QAM MIMO symbols, as in [8], [9]. For the simulation, 2 and 4 receive antennas have been considered. The decoding is performed by the Viterbi's algorithm.

The total transmitted power is normalized, i.e. the sum of the powers of the transmitted signals is equal to 1 . Thus, to normalize the total transmitted power of 16-QAM STTCs, the MIMO signals must be multiplied by 0.4472 if $n_{T}=2$, by 0.3651 if $n_{T}=3$ and by 0.3162 if $n_{T}=4$. For 64-QAM STTCs, the MIMO signals must be multiplied by 0.2182 if $n_{T}=2$ and by 0.1781 if $n_{T}=3$.

The performance of 16-QAM STTCs with 2 transmit antennas given in Table II is presented in Fig. 2 for 2 and 4 receive antennas. The new 16-QAM 16 states code and the corresponding WYCK's code have the same performance, but the research time to find the new code is much lower. The performance of Hong's code is slightly worse than the new 
corresponding code because it has been designed with the rank and determinant criteria. The Hong's code is optimal with one receive antenna.

Fig. 3 shows the performance of the new codes proposed in Tables II and III in the case of 2 and 4 receive antennas.

Fig. 4 shows the performance of the new codes proposed in Table IV in the case of 2 and 4 receive antennas.

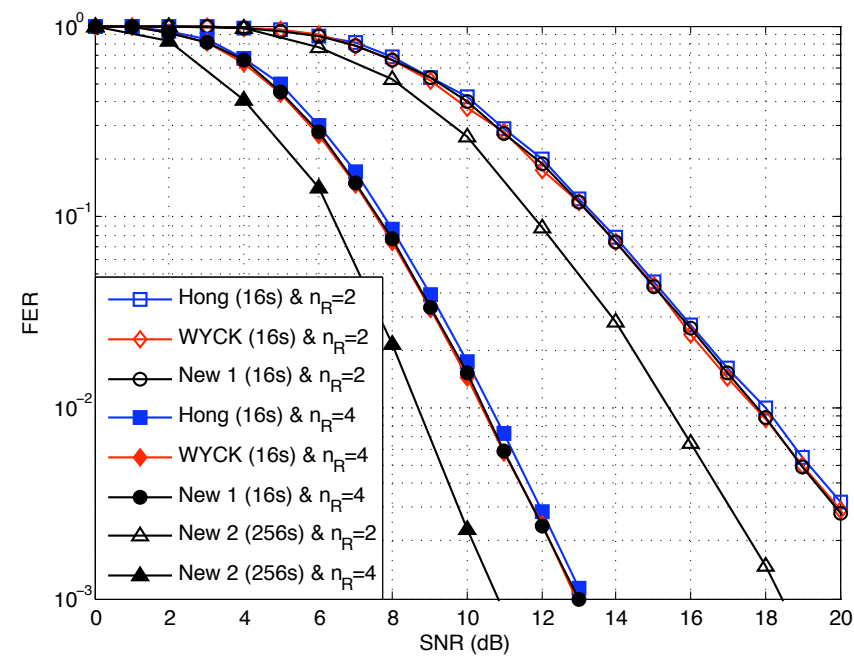

Fig. 2. 16-QAM 16/256 states STTCs with 2 transmit antennas and 2 \& 4 receive antennas

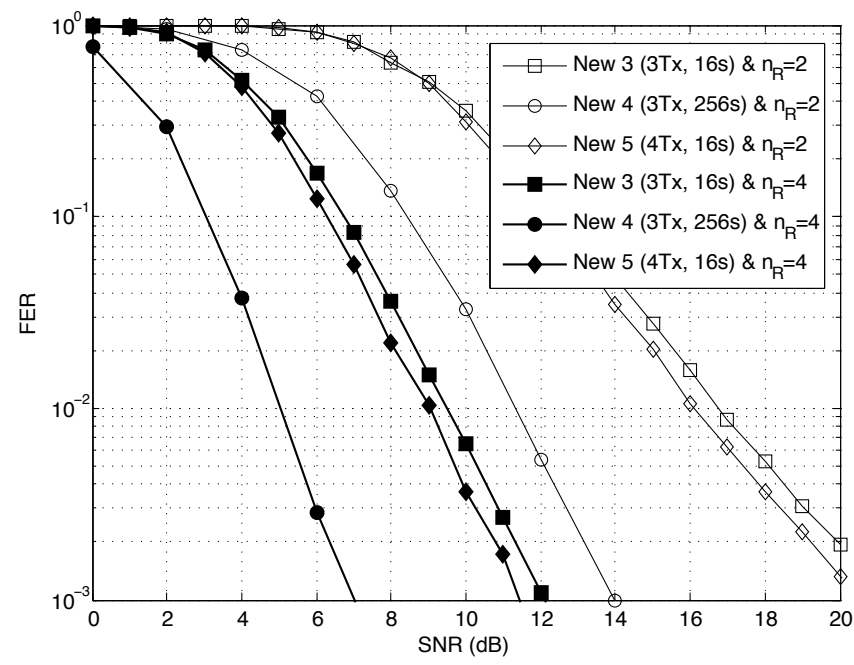

Fig. 3. 16-QAM 16/256 states STTCs with $3 \& 4$ transmit antennas and 2 \& 4 receive antennas

\section{CONCLUSION}

The design of the QAM STTCs has been considered in this paper. The difficulty to find the best QAM STTCs is the number of codes which must be analyzed. For example, there are several billions of 16-QAM 16 states STTCs with 2 transmit antennas. Thus, for the exhaustive search, the time to find the best codes among all the possible codes is critical, especially when the number of transmit antennas or/and the

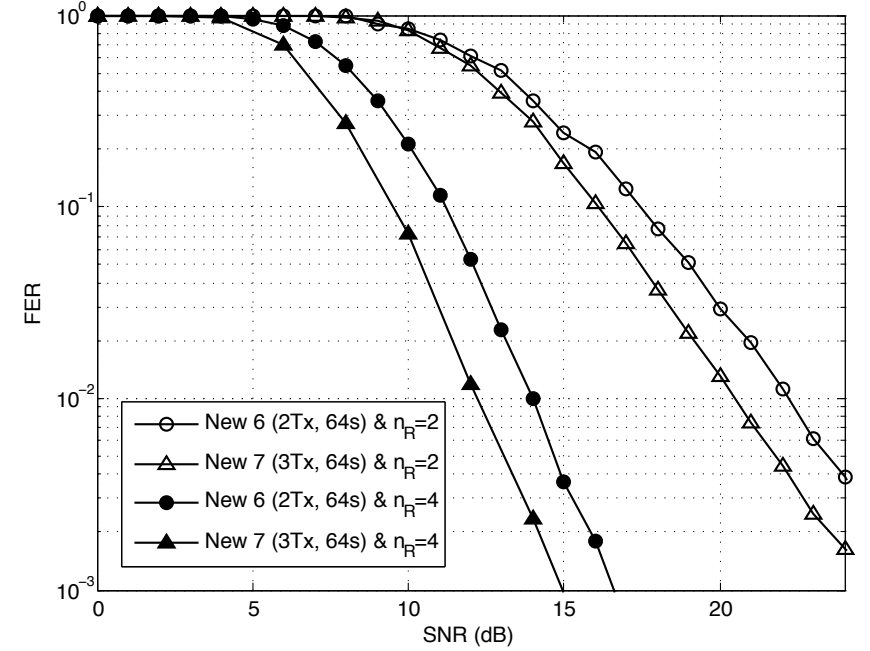

Fig. 4. 64-QAM 64 states STTCs with $2 \& 3$ transmit antennas and $2 \& 4$ receive antennas

number of states increase. Therefore, the previous publications proposed only 16-QAM codes with two transmit antennas. In this paper, a new efficient method to generate optimal QAM STTCs is presented. This method is based on the Euclidean distance decomposition and the division into blocks of the generator matrix. It allows to reduce significantly the search time to obtain the optimal codes. Thus, via this new method, 16-QAM STTCs with more than 2 transmit antennas and 64-QAM STTCs with 2 and 3 transmit antennas have been proposed for the first time.

\section{REFERENCES}

[1] V. Tarokh, N. Seshadri, and A. Calderbank, "Space-time codes for high data rate wireless communication: Performance criterion and code construction," IEEE Transactions on Information Theory, vol. 44, no. 2, pp. 744-765, Mar. 1998.

[2] D. Ionescu, "New results on space-time code design criteria," in Proc. IEEE WCNC 1999, 1999, pp. 684-687 vol.2.

[3] Z. Chen, J. Yuan, and B. Vucetic, "Improved space-time trellis coded modulation scheme on slow fading channels," Electron. Lett., vol. 37, no. 7, pp. 440-441, Mar. 2001.

[4] Z. Chen, B. Vucetic, J. Yuan, and K. L. Lo, "Space-time trellis codes with two, three and four transmit antennas in quasi-static flat fading channels," in Proc. IEEE ICC 2002, vol. 3, May 2002, pp. 1589-1595.

[5] C. Liao and V. Prabhu, "Improved code design criteria for space-time codes over quasi-static flat fading channels," in Proc. IEEE SPAWC 2005 , June 2005, pp. 7-11.

[6] D. Aktas and M. Fitz, "Distance spectrum analysis of space-time trellis-coded modulations in quasi-static rayleigh-fading channels," IEEE Transactions on Information Theory, vol. 49, no. 12, pp. 3335 - 3344, Dec. 2003.

[7] Y. Liu, M. Fitz, and O. Takeshita, "A rank criterion for QAM space-time codes," IEEE Transactions on Information Theory, vol. 48, no. 12, pp. 3062-3079, Dec 2002.

[8] A. Wong, J. Yuan, J. Choi, S. R. Kim, I.-K. Choi, and D.-S. Kwon, "Design of 16-qam space-time trellis codes for quasi-static fading channels," in Proc. IEEE VTC 2004-Spring, vol. 2, May 2004, pp. 880883 Vol.2.

[9] Y. Hong and A. Guillen i Fabregas, "New space-time trellis codes for two-antenna quasi-static channels," IEEE Transactions on Vehicular Technology, vol. 56, no. 6, pp. 3581-3587, Nov. 2007.

[10] G. Ungerboeck, "Channel coding with multilevel/phase signals," IEEE Transactions on Information Theory, vol. 28, pp. 55-67, Jan. 1982. 